\title{
POLÍTICAS PÚBLICAS SOBRE VIOLENCIA Y TELEVISIÓN: UN ENFOQUE DESDE VARIAS DISCIPLINAS Y ALTERNATIVA DE CONSTRUCCIÓN SOCIAL EN TRABAJO SOCIAL
}

\section{Emilia Morales Lara ${ }^{1}$}

\section{Resumen}

En este artículo se explora la forma como se ha abordado hasta el presente el tema de la violencia en la televisión y su impacto en la niñez, desde el Trabajo Social y desde otras disciplinas. Se centra la atención en la forma en que cada profesión ha incidido en la estructuración de políticas públicas sobre el tema en los últimos años. Se establecieron los puntos de vista más reconocidos sobre los que la psicología, la comunicación social, la sociología, la economía, y el derecho actúan, y los elementos que de estas ciencias puede recoger el Trabajo Social para su actuar. Se observó el gran potencial de lecciones para la profesión, y la forma como estas profesiones enriquecen el aporte hacia la proposición de modelos de prevención de la violencia, de atención a situaciones existentes, y la organización comunitaria y la construcción social de modos de vida dignos.

Descriptores: violencia en la televisión, política pública, construcciones sociales de diferentes disciplinas.

\section{Abstract}

This article explores the topic of violence in television and its impact on childhood, and how this has been approached until the present, by Social Work and other disciplines. The attention is focused on the way each profession has impacted in the structure of public policy about the mentioned issue in the last

\footnotetext{
${ }^{1}$ Estudiante del Programa Doctoral de la Escuela Graduada de Trabajo Social Beatriz Lassalle, Universidad de Puerto Rico en Río Piedras.
} 
years. It analyzes the points of view of psychology, communications, sociology, economy and law. It gathers the elements that Social Work can use for its action. The great potential of lessons for the profession, the way these professions enrich the prevention of violence, the attention to existent situations, the community organization, and the social construction are observed to construct appropriate models of life for our populations.

Keywords: violence in television, public policy, social constructions from different disciplines.

\section{Introducción}

Es reconocido el impacto que tienen los programas y mensajes televisivos en los comportamientos individuales y colectivos de las personas, y especialmente de los niños. La incidencia de los programas de ficción y los noticieros presentados en la televisión en las últimas décadas ha sido ampliamente reconocida y estudiada por investigadores en todo el mundo, con una fuerte concentración en los aspectos atinentes a la violencia (Piaget, 1970; Walsh \& Gentile, 2001). Son frecuentemente citadas las características de productos cuidadosamente diseñados para lograr su comercialización como mercancías, además del uso abundante de estereotipos o "representaciones sociales, institucionalizadas, reiteradas y reduccionistas" (Ferrés, 1996), para que el niño asimile bajo formas atractivas, convincentes y aceptables conceptos como la discriminación, el racismo, la xenofobia, el sexismo, y la violencia física y armada como única solución para los conflictos (Maturana, 2002). Su resultado es que el niño los consume y asimila la visión de la realidad que los productores quieren transmitir: La guerra y la violencia se presentan como componentes esenciales de la realidad humana, y no solo son inevitables, sino necesarias "para poner de nuevo el mundo en un estado de feliz equilibrio" (Ferrés, 2002).

Este reconocimiento a su vez ha impulsado en muchos países y a través de movimientos internacionales, presiones sociales y de grupos específicos para el establecimiento de políticas públicas que garanticen (viabilicen, desarrollen, establezcan, protejan...) la calidad de la televisión, el mejoramiento de las formas de convivencia, y la preservación de la salud mental de la población más vulnerable, especialmente de la infantil.

Para el trabajador y trabajadora social esta temática no es ajena. En muchas ocasiones se encuentra con entornos sociales o políticos violentos en los que 
deben subsistir o sobrevivir la niñez. Además, estos niños y niñas no tienen a su alcance patrones de conducta o ejemplos sociales o individuales que les muestren las condiciones en las cuales se desarrolla una vida digna, y tampoco encuentran herramientas sociales con las cuales hacer frente o sobrevivir dignamente a los hechos y efectos de la violencia. Se encuentra también con comunidades enteras inmersas en el círculo vicioso de la violencia, sin perspectivas ni modelos convincentes de convivencia que les permita una construcción socialmente válida de modelos de vida con tolerancia, entendimiento y aceptación del otro y de las diferencias.

Sin embargo, la forma como resulta posible actuar desde el trabajo social para la consecución de políticas públicas acerca de la televisión no ha sido estudiado en forma sistemática. Los diferentes modelos de atención a la problemática tienden a realizar acciones de atención terciaria predominantemente, y escasas acciones preventivas y de construcción comunitaria. Vemos los resultados y tratamos de incidir, pero poco hacemos en función de la prevención y de la búsqueda de protecciones sociales integrales ante el fenómeno. El profesional de trabajo social se ha quedado enclaustrado en el paradigma de utilizar estrategias de intervención encaminadas a la atención individual de personas con patologías sociales y emocionales (Canino y Guardiola, 1995). Las problemáticas que se desprenden de la generalización de conductas sociales disfuncionales no se resuelven en su origen, como políticas públicas y como conciencia colectiva, sino en sus efectos, en la mayor parte de los casos como atención a situaciones ya creadas e incluso avanzadas que dificultan enormemente su solución.

El presente trabajo pretende involucrar la profesión de trabajo social en el debate sobre la importancia de incidir en políticas públicas que minimicen el efecto nocivo de los medios de comunicación, especialmente la televisión, en la conducta violenta de los niños, y garanticen una televisión con visiones de dignidad y portantes de herramientas para la solución de conflictos en nuestras sociedades. Para ello resulta importante reconocer la forma como otras profesiones, como la psicología, la comunicación social, la sociología, la economía y el derecho tienen su propia percepción y enfoque sobre la necesidad de incidir en tales políticas, y recoger de ellas lecciones útiles para el desenvolvimiento del trabajo social. 


\section{El espectro de las políticas públicas en cuanto a la violencia en la televisión}

A pesar que en este trabajo se analizarán los enfoques desde el punto de vista de cada profesión, es importante considerar la existencia de escuelas y tendenciasdel pensamiento, que han orientado las distintas aproximaciones actuales a las políticas públicas en materia de violencia en televisión. Como se observará más adelante, tales tendencias penetran profundamente en las estructuras profesionales para construir estructuras de explicación y por tanto de respuesta al fenómeno, que caracterizan su centro de atención. Estas escuelas pueden ser vistas, para efectos de la presente ponencia, desde dos parámetros: a) según el grado de aceptación de la existencia de la televisión como fenómeno inductor de violencia en los niños, y b) según la tendencia a concentrar las acciones de respuesta en defensa de los niños, en manos de alguno de los actores dentro del proceso de comunicación.

De acuerdo con la forma como tal construcción se realiza, es posible derivar cuáles enfoques tienden a sostener y garantizar el status-quo, y cuáles constituyen una opción alternativa que modifique la actual fuerza casi monopólica de los medios, que se observan como empresas rentables y formadoras de visiones unificadoras y justificadoras de las inequidades del mundo que dirige tal poder. Según el primer parámetro (el grado de aceptación de la existencia de la televisión como fenómeno inductor de violencia en la niñez), es posible observar una amplia gama de actitudes. La primera asume que la televisión es un medio neutral (CAC, 2002), que simplemente presenta situaciones reales o elementos para la diversión de las personas. Para ésta, es necesario promover una actitud de apertura, diálogo y descubrimiento de la cultura propia y las culturas ajenas. Una segunda actitud cuestiona el medio en su dimensión de constructor de realidad. Considera que actúa con libertad, pero en la medida en que transmite matrices de representaciones, funda la visión del mundo del receptor y, a veces, su acción en el mundo. Esta acción depende de la capacidad del televidente para preguntarse dónde está la verdad, y si existe una verdad que no sea construida por tales representaciones. La más radicalmente opuesta a la presencia de la televisión en nuestras vidas tiende a rechazar individual o colectivamente esta tecnología, al verla como vehículo para la intrusión de culturas extranjeras, poniendo en peligro la cultura y los valores propios, así como las estructuras pacíficas de coexistencia entre las personas (Ollivier, 1997). 
Por otro lado, desde la óptica del actor responsable de activar los procesos de defensa de la salud mental de la niñez, también existen tendencias o actitudes claras. En primer término, existen investigadores, investigadoras y profesionales que abogan por el papel protagónico en manos de las personas consumidoras del producto televisivo. De acuerdo con NASP (2002) y CAC (2003), las actividades tendientes a defender a nuestra niñez de los efectos nocivos de la televisión, recaen fundamentalmente en manos de sus progenitores, profesoras y profesores, aunque también es importante crear una conciencia en el teleaudiente, que le genere competencias para reconocer y defenderse de los efectos nocivos del medio, bajo lo que Penalva (2002) denomina 'alfabetización en medios' ("media literacy"). El Estado en algunos caos se observa como un simple garante de las plenas libertades en la sociedad, y desde esta perspectiva se considera su intervención como una indebida intromisión en los asuntos relativos a uno de los derechos más defendidos: la libertad de expresión, y por lo tanto de comunicación y de prensa (CIP, 1994). Como máximo, el papel del Estado debe centrarse en la atención a niñas y niños afectados o en riesgo de afectación por conductas violentas, a través de agencias especializadas en las cuales los sicólogos y los trabajadores sociales juegan un papel central (Singer, Slovak, Frierson \& Cork, 1998).

En segundo lugar, se propende por el papel determinante de colectivos, sea comunidades o grupos de interés, en las acciones que permitan controlar o evitar los efectos nocivos de la televisión en los comportamientos violentos de niños y niñas. Bajo esta tendencia, se proponen acciones que hagan que las comunidades presionen la existencia de instrumentos de regulación pública sobre los medios, especialmente sobre los programas de ficción y los noticieros (Grupo De Defensa Psicológica, 2004). También que las comunidades desarrollen sus propios programas y medios de comunicación con criterios de desarrollo comunitario y valoración de la paz y la convivencia (Gordon \& Holguín, 2005). Otros colectivos propuestos como actores son las asociaciones de profesionales de la comunicación y periodistas. Desde esta óptica, las asociaciones de comunicadores pueden ejercer presiones para que los gobiernos legislen y regulen los medios televisivos, pero también pueden comprometerse y aplicar sus esfuerzos para construir una televisión de calidad, con parámetros de dignidad y respeto a la integridad y salud mental de niños y niñas. Desde esta perspectiva, la acción mínima a exigir a las programadoras de televisión es la denominada autorregulación, según la cual los programadores establecen sus propios lineamientos y restricciones al diseñar el producto televisivo 
(códigos de ética, reglamentos internos, etc.), y como expresión directa hacia el televidente, establecen unos parámetros recomendados de consumo que tienen que ver principalmente con la edad mínima considerada adecuada para ver los programas, y los elementos presentes en el programa, en términos de lenguaje, sexo y violencia (The TV Parental Guidelines, 2005).

En tercer término, existe la opinión que indica la importancia de un papel estatal como regulador del sector de la televisión y los medios de comunicación. Sus opciones son el establecimiento legal de orientaciones a los productores, la reglamentación de limitaciones a la otorgación de licencias y permisos de operación, y la determinación de prohibiciones en términos del diseño y presentación de contenidos. Estas opciones de intervención son ampliamente reconocidas y utilizadas en sus matices en muchos países. Estos criterios de intervención buscan respetar la plena libertad de expresión en los medios y la libertad de empresa, guardando para ciertos momentos la potestad de intervención, para evitar los efectos nocivos del producto televisivo a la comunidad, o los valores compartidos socialmente (Lara, 2004). El desarrollo de estos criterios de acción exige a su vez la existencia de organismos públicos encargados de la vigilancia y el control.

Finalmente, se encuentra la tendencia a que sea el Estado quien produzca y emita los programas de televisión, sea a través de propiedades parciales como productor a la par con otros productores, o como propietario de canales y medios de comunicación. Desde esta perspectiva, el papel del Estado es directo, tanto en el diseño como en el establecimiento de las condiciones de presentación y difusión de contenidos y mensajes, lo que hace que su poder y presencia sea directo, y que su responsabilidad esté directamente relacionada con el tipo de producciones que emita (Penalva, 2002).

\section{El aporte de las ciencias y sus profesiones a las políticas públicas sobre violencia y televisión}

\section{Aportes desde la Psicología}

Desde el punto de vista de la psicología existe un reconocimiento generalmente aceptado acerca de la influencia que tiene la televisión en la conducta violenta de las personas, y especialmente de niños, niñas y jóvenes. Tanto la psicología constructivista como la social han realizado importantes avances en este campo, 
desde Bandura y Walters en 1963. Sin embargo, no todas estas concepciones coinciden en cuanto a la forma como debe estructurarse la política pública y como el Estado debe intervenir para regular o proteger a la población con respecto al fenómeno.

La teoría de la asociación cognitiva propuesta por Berkowitz (1984-1986) plantea que los contenidos agresivos sugeridos por una película violenta pueden activar otras ideas relacionadas semánticamente, aumentando así la probabilidad de que el observador tenga ideas agresivas que algunos individuos no pueden controlar. Por su parte, investigadores como Penalva (2002), estructuran acciones para el fortalecimiento del niño o niña para que haga un consumo inteligente de la televisión. Algunos estudios y propuestas de política pública (WFMH, 2004), emprenden acciones de fortalecimiento y prevención a través de publicaciones y realización de actividades educativas y formativas.

También la psicología social aboga por la construcción social de comunidades activas, críticas y constructoras, capaces de detectar y poner en evidencia la actuación irregular de la televisión y la radio, identificando el carácter premeditado de su comportamiento y el objetivo socio-político que persiguen, proponiendo la definición de políticas a los medios del estado y alternativos, sugerir la creación de espacios televisivos de análisis de contenido a programas, publicidad y propagandas, desarrollar programas de formación crítica de la audiencia (Grupo de Defensa Psicológica, 2004).

\section{Aportes desde la Comunicación Social}

Los comunicadores y comunicadoras, también reconocen la existencia de la relación entre violencia en televisión y comportamientos violentos en la sociedad. Por ejemplo, Gloria Tristani, ex comisionada de la Comisión Federal de Comunicaciones en Puerto Rico, reconoció en 1998 el nivel de violencia en la televisión. Declaró que: "un niño en promedio mira aproximadamente 25 horas de televisión por semana. Para cuando hayan completado la escuela primaria, los niños habrán mirado cerca de 8.000 asesinatos y 100.000 actos de violencia". Sin embargo, ésta influencia no aparece bajo la forma de una imposición directa, dictatorial o imposibilitadora para el niño o niña ni para sus progenitores. Existen principalmente dos mecanismos (que operan en el conjunto de los campos comunicacionales) mediante los cuales se logra inculcar en las mentes de las personas la violencia bajo formas convincentes, aceptables, e incluso deseables. 
El primero de esos mecanismos es el de la consecución de la hegemonía a través de la negociación. La imposición directa de las ideas y los intereses de una persona o grupo genera rechazos en las sociedades modernas que cuentan con abundantes poblaciones con capacidades sociales y conocimientos que han elevado como valores fundamentales la libertad, la equidad y la justicia. La hegemonía ("la capacidad que tiene un grupo social de ejercer la dirección intelectual y moral sobre la sociedad..." Mattelart y Mattelart, 1997), se consigue mediante negociaciones que hacen que las clases subalternas reconozcan como suyos los intereses de las clases hegemónicas (Sepúlveda, H., 2005). "La dominación se negocia hasta consentir"' (Sepúlveda, H., 2005). Así, la dominación se cumple tanto en el nivel consciente como en el inconsciente. Por ello el papel de los medios de comunicación es tan importante y reconocido. El segundo mecanismo es el de la seducción. Este mecanismo tiene importante alcance, pues apela a la interpelación lúdica, a la espectacularidad, y en general a todos los elementos que generan seducción en sus distintas acepciones: persuadir suavemente para algo, atraer físicamente a alguien con el propósito de obtener de él una relación, o embargar o cautivar el ánimo (Sepúlveda, 2006).

Para Barbero (2002), en América Latina se presenta una paradójica actitud en los medios: pesimismo frente a la visión social de las macro tendencias a la par con fascinación frente a las maravillas tecnológicas. Converge la fascinación tecnológica con el realismo de lo inevitable. A la par, aparecen otras paradojas: la convivencia del derroche estético de los centros comerciales con las condiciones insalubres e inhabitables de los barrios de invasión, la opulencia comunicacional con el debilitamiento de lo público, la más grande disponibilidad de información con el palpable deterioro de la educación formal, la continua explosión de imágenes con el empobrecimiento de la experiencia, la multiplicación de signos y el déficit de sentido.

La convergencia entre sociedad de mercado y racionalidad tecnológica "disocia la sociedad en sociedades paralelas: la de los conectados a infinita oferta de bienes y saberes, y la de los excluidos cada vez más abiertamente tanto de los bienes como de la capacidad de decidir". (Barbero 2002). Estos elementos conllevan fuerzas desintegradoras: La desmitificación de las tradiciones y las costumbres que permitían confianza, el arraigo de la violencia, la erosión de los mapas cognitivos (desprecio por el saber local y nacional), llevando a otra crisis: la de los mapas ideológicos. El resultado es contundente: dentro de este cambio lo cultural se centra en lo audiovisual, y particularmente en la televisión. 
La televisión se constituye en dispositivo radicalizador del desanclar que produce la modernidad, redefiniendo las jerarquías que normaban la cultura y también sus modalidades, niveles y lenguajes. Por otro lado, comunicación es también sinónimo de lo que manipula y engaña, lo que nos desfigura políticamente como país y nos destruye culturalmente como pueblos (Barbero 2002).

\section{Aportes desde la Sociología}

Los sociólogos asumen diversos enfoques con respecto a influencia de la televisión y la violencia en el comportamiento de la niñez. Por ejemplo, Ferrés (1996) considera que el papel de la televisión como agente socializador se fundamenta en dos hechos: a) el porcentaje tan alto de tiempo libre que le dedican las personas, b) su capacidad de simular la vida real y crear situaciones fantásticas. Estrada (1995) afirma que la influencia de la televisión es un fenómeno según el cual se atribuye valor social al saber, según la calidad y oportunidad de su presentación. Si el proceso informativo es continuo y permanente, dificulta la profundización en los temas, produciéndose la transformación del conocimiento en información.

Alrededor del tema de la política pública existen también varias tendencias. Para quienes abogan por una concepción positiva de la televisión y una construcción social del saber colectivo, se considera que el receptor no es pasivo, que la cultura no es sólo alta cultura y que la televisión no es conspirativa, lo que elimina el peligro de la amenaza embobante de la TV. En lugar de "masa" se habla de "públicos" y en lugar de mensajes de alienación existen "contextos de recepción y procesamiento" (Openhayn, M. 2001). Desde esta perspectiva, el peso predominante en el control de contenidos corresponde tanto al teleaudiente y su familia, como al productor, quien debe conocer y respetar tanto el contexto cultural en el que se desenvuelve el espectador, como la cultura que moldea en le mismo. Bajo estas ideas, la TV no puede pretender ser exclusivamente educadora en sentido iluminista /ilustrado, propagandista de las ideologías de gobierno o las razones de Estado, paternalista, e inviable económicamente.

Para algunos investigadores como Castells (1996), con la introducción de la sociología de la cultura, los medios de comunicación son entendidos como mediadores entre receptores y realidad, los mensajes no son transparentes ni unívocos y como instituciones. Bunge (1995) indica que los medios de comunicación son significantes de una realidad, por lo cuál es necesario 
comprender su funcionamiento sociocultural, económico y político. Tal comprensión lleva a entender la responsabilidad social y el papel que juegan los medios de comunicación y en especial la televisión en la formación de contenidos, culturas y contextos para la acción y decisiones de las personas. De allí la importancia de la regulación estatal, cuya orientación apunta a garantizar que estos contextos se respeten y se apliquen sin generar daño a los telespectadores.

\section{Aportes desde la Economía}

La economía tiende a abordar el tema de la política pública en forma central, ya que sus diferentes escuelas se diferencian en gran medida por el papel atribuido al Estado en la producción, circulación y distribución de la riqueza. Así, las tendencias existentes con respecto a las políticas públicas en el tema de televisión y violencia se diferencian claramente. En un extremo, se encuentra la tendencia neoliberal, según la cual todos los bienes y servicios deben ser prestados por los particulares, bajo claros criterios de mercado. De acuerdo con esta tendencia, en el mercado de televisión existe completa libertad de decisión por parte del consumidor. Éste a su vez cuenta con información completa, suficiente y sin restricciones para realizar sus elecciones, y de acuerdo con su capacidad de pago selecciona los programas y contenidos a los que tiene igual derecho de acceso que el conjunto de la ciudadanía. Se supone a su vez que no existe monopolio y por tanto no existe el riesgo que algún medio tenga intenciones ni interés en moldear la opinión y conducta de las persona según su interés comercial (Osborne, 1995). Según esta teoría, debe operar la 'mano invisible', es decir, la no presencia del Estado en tal mercado, dejándolo operar según las decisiones y a los precios que establezcan los oferentes y demandantes. Siempre los televidentes tendrán la televisión que desean y los programadores tendrán las ganancias merecidas según coincidan con las preferencias de los consumidores.

En el otro extremo se encuentran los marxistas, quienes propenden por el control estatal de la televisión. Sin embargo, la teoría marxista no puede considerarse en su estructura tradicional, pues ha evolucionado hasta convertirse en una teoría completa de regulación. Para ellos, la violencia en la televisión es parte del mercado, y por lo tanto se ve por parte de los programadores como connatural a los criterios de aumentar el "rating" para que sea mucho más productivo en términos económicos. El criterio de la ganancia predomina, y a la vez forma el pensamiento que resulta conveniente a las grandes corporaciones nacionales e 
internacionales, con cifras de facturación gigantescas, vínculos empresariales estrechos con otras grandes empresas y grupos financieros, y afinidades con partidos políticos o grupos de presión (Aznar, 2005). Esto le da a la televisión un doble carácter: un medio de influencia, y una fábrica de hacer dinero. Así, las garantías de adecuación de contenidos, desarrollo de identidad nacional y establecimiento de bases de respeto hacia la población infantil pasan por la estructuración de fuertes regulaciones a los medios televisivos o por la propiedad estatal de programas, programadoras, o canales de televisión.

Como tendencia intermedia entre las descritas se encuentran los economistas institucionales o neoinstitucionales, quienes consideran que la televisión es efectivamente un mercado, pero al contrario de los neoliberales, observan que su operación es imperfecta. La información no es completa ni está al alcance de todos los consumidores, existen fuertes tendencias al monopolio, la información es asimétrica (más conoce del medio quien lo ofrece que quien lo consume), la formación de precios no es transparente (su apariencia es gratuita o de bajo costo, pero en realidad la publicidad la hace altamente costosa y de cobertura social general). Estos factores hacen necesaria la presencia del Estado a través de regulaciones (North, 1990). La mano invisible no opera, y es necesaria la acción estatal a través de los diferentes mecanismos de regulación, tales como la autoregulación, la orientación pública a los productores, las limitaciones o prohibiciones con respecto a la otorgación o renovación de licencias, e incluso la creación estatal de programas, programadoras o canales de televisión (Coase, 1994).

\section{Aportes desde el Derecho}

El enfoque fundamental del derecho con respecto al tema de los medios de comunicación es eminentemente formal, enraizado en las bases de la libertad de las sociedades democráticas de tipo republicano. En ellas se establece que la sociedad tiene derecho a una información fundada en la verdad, la libertad, la justicia y la solidaridad. Por esta razón, los estudiosos del derecho en general tienden a enunciar su reticencia a regular y en general a legislar frente a este poder. Sin embargo, dado que en la mayoría de países se habla de una información versada en la verdad, por lo que no existe regulación frente a la misma. Es de conocimiento publico que esa verdad es relativa porque hay verdades que obedecen más a criterios de poder y ganancia. Esta paradoja es más clara en el modelo norteamericano, muy respetuoso del mercado y de la autocensura, que en el modelo europeo, en el cual el estado siempre ha tenido 
un papel predominante desde principios del siglo XX. Incluso, en tales países el Estado acompañó y orientó el desarrollo de los medios de comunicación mediante acciones y reglamentaciones muy fuertes, los cuales se han ido reduciendo en las últimas décadas, acercándose más al modelo de los Estados Unidos. Esta misma tendencia se aprecia en América Latina, en la cual la televisión se inició y desarrolló con el impulso directo del Estado y bajo la regulación y control directo de éste (Brunner y Catalán, 1994).

El derecho se ocupa también de una clave de análisis para el proceso de la comunicación social: Desborda el derecho a informar lo que la gente necesita saber, y alcanza al método o procedimiento para la obtención y recopilación de los datos por parte de los profesionales de la información, y a la forma que se da a esa información para su difusión, tanto en el campo de las noticias como en el de la producción de ficción. La justicia, por lo tanto, no aplica sólo al derecho del receptor, si no que también a las personas que son fuentes de información y a aquellas que por sí mismas constituyen noticia. También a ellas les es debido respeto a su dignidad y a su honra. Cuando no se respeta a las personas, su vida privada, o su honra, se está también faltando a la justicia derecho a recopilar, procesar, difundir y recibir información, tal como ocurre en los programas de farándula y en los "Reality Shows", en los que los personajes reales con mostrados como ejemplos de violencia, en condiciones denigrantes y como ejemplos de comportamiento social a los jóvenes (ONU, 2004).

\section{Lecciones para el Trabajo Social}

A pesar de la existencia de colegas preocupados por el tema, y que han escrito importantes documentos al respecto (Penalva, 2002), como profesión es poco lo que se ha estudiado o abordado éste tema, y creo que es hora de que la profesión se involucre en este debate, ya que no es sólo competencia de los comunicadores: es competencia de toda profesión que se sienta comprometida por los 'asuntos sociales'. Es una "responsabilidad social, a partir del compromiso libre y riguroso de los agentes sociales, es decir de todos nosotros. Una responsabilidad que debe ser tanto individual como colectiva" (Aznar, 2005).

No es oportuno propugnar un desarrollo de estudios independientes, realizados por profesionales de trabajo social, con el fin de desarrollar una visión propia y excluyente sobre el tema, sustituyendo los conocimientos y alcances ya 
establecidos sobre el mismo. Sin embargo, la investigación sobre violencia y televisión desde la perspectiva del trabajo social tiene gran importancia, como una necesidad de aporte desde nuestra experiencia y punto de vista (cercanos a las vivencias de la población), reconociendo los aportes ya existentes por las diferentes disciplinas. A su vez, el análisis de las políticas públicas sobre medios de comunicación y violencia adquieren relevancia en nuestros países, en los cuales la incidencia de este factor se ha convertido en endémica y en algunos casos epidémica, y sobre los cuales la capacidad de presión o acción por parte de los trabajadores sociales no es reconocida.

Así, de las distintas ciencias y vertientes del conocimiento, es posible obtener una serie de lecciones valiosas para el trabajo social, que a la vez orientan posibles campos de investigación sobre el área. Es necesario "construir un nuevo pacto social fundante", como lo dice Primavera (1999). Y hay que hablarlo y construirlo al interior del colectivo de trabajo social, para hablar con el exterior. Debemos definir también cómo pensamos, desde qué lugar lo hacemos, hacia dónde nos dirigimos y qué consecuencias tiene este conjunto de reflexiones para nuestra práctica profesional. En este sentido se busca explorar el proceso de construcción que apunte al diseño de nuevas prácticas sociales entre los distintos actores sociales.

Las poblaciones que sufren condiciones de pobreza, desigualdad y discrimen tienen cada vez menos alternativas de moldeamiento social positivo ante la presión para la absorción de estos moldes, bajo la monopolización de los medios de comunicación comerciales y la desaparición de los medios tradicionales de moldeamiento social como la educación formal. A la vez, al configurarse los medios de comunicación de masas como un 'cuarto poder', se convierten en mediadores y moldeadores entre las políticas públicas y la población objeto de las mismas. En la mayor parte de nuestros países, el Estado ha dejado a la libre competencia el moldeamiento real de valores, sentimientos y apreciaciones de la población, permitiendo que esa libertad comercial se asuma como 'libertad de prensa'. El profesional de trabajo social debe jugar un papel activo en la formación social y en el impulso a la responsabilidad estatal sobre la conformación de nuestra sociedad.

Dada la problemática social de los países de América Latina, en los cuales se encuentran los mayores problemas ocasionados por la desigualdad social, se requiere que se desarrollen nuevas estrategias de intervención como la regulación 
de los medios masivos de comunicación tendientes a su aporte a la dignidad de vida y la educación a comunidades y grupos utilizando los diversos medios masivos. A la vez debemos impulsar nuevas estrategias de difusión y moldeamiento de conductas socialmente dignificantes y culturalmente constructivas, que presionen políticas públicas también dignificadoras, que lleguen a mayor cantidad de población y que logren transformar sus condiciones de vida.

En la construcción de un modelo de comunicación en este sentido, el trabajo social puede apoyar en la configuración de sistemas de autorregulación que signifiquen funcionalidad, solidaridad entre todos los elementos del sistema, contacto permanente entre los mismos, equilibrio y retroacción, circulación constante. Autorregulada será entonces una sociedad de relaciones complejas y móviles que harán de cada uno un nudo en el circuito de la comunicación incesante.

En ese contexto se hace necesaria la existencia de un modelo de acción para la construcción social en el cual los trabajadores sociales podamos incidir hacia la creación o profundización de políticas públicas que dignifiquen la vida humana. Las bases de este modelo son el crear redes de apoyo para generar políticas reguladoras, en las que se incluya trabajo con las autoridades y más altas esferas de la política pública (cabildeo con legisladores y directivos de agencias públicas, especialmente de educación, salud y familia); así como la organización decidida de grupos de base en las comunidades, (padres y madres de familia, profesores y profesoras) escuelas de trabajo social, facultades de comunicación, con el aporte de las más diversas profesiones, en torno a que televisión queremos en nuestros países.

Otra recomendación es la de involucrar a trabajadores y trabajadoras sociales y a la profesión en su conjunto en este debate, con el fin de obtener información de primera mano sobre el fenómeno, recoger opiniones y puntos de vista acerca de los efectos observados y las posibilidades de modificación de la política pública, y para apoyar profesionalmente el desarrollo del proceso de presión y divulgación sobre los cambios propuestos. Es importante también, generar presión social para buscar que la televisión se enfoque más hacia la promoción de estilos de vida liberadora, participativa y al reconocimiento cultural, y hacia la prevención de situaciones y conductas que atenten contra la dignidad y la calidad de vida de las familias y población en general. En este contexto se 
recoge la propuesta de lucha contra la injusticia, la discriminación la exclusión social y cultural (Beltrán, 2000).

El modelo debe incluir el trabajar con las pequeñas comunidades mismas y en los centros en los que se desenvuelve el profesional de trabajo social cotidianamente. Es importante además, el apoyar a las organizaciones sociales (sindicatos, asociaciones campesinas, juntas vecinales, organismos no gubernamentales, etc.; y poner énfasis prioritariamente sobre la comunicación para la salud, el saneamiento, la nutrición y la demografía.

Las siguientes pueden considerarse como externalidades positivas del modelo propuesto:

- Relaciones más fraternas entre pares

- Mayor comunicación en la familia

- Menor agresión en las relaciones intrafamiliares

- Estímulo a valores de solidaridad

- Mayor conciencia, capacidad de crítica

- Incidencia positiva en la solución de varios problemas sociales

- Acción preventiva de diversas áreas de intervención

- Mayor información instructiva y educativa a la población

- Fomento a la organización comunitaria, y fortalecimiento social

- Disminución en la incidencia criminal

Una futura generación con elementos conceptuales para presentar alternativas diferentes a la violencia para resolver conflictos. Con estas condiciones aplicadas, este será un cambio 'pacifico pero revolucionario'. Un cambio 
silencioso pero efectivo, un cambio sin dinero pero con riqueza humana. Como lo expresa Rozas (1993):

En este marco es necesario repensar el papel de a Política y el fortalecimiento de a sociedad civil. Frente a la crisis de las comprensiones totalizadoras de la realidad se hace necesario crear nuevos conceptos y darles significado a los existentes...nos encontramos con nuevos problemas que hacen compleja la realidad social.

Guardiola (1993) nos dice que en Puerto Rico:

Vemos cómo la miseria y la alineación de la sociedad moderna se ha desdoblado dando nuevas pobrezas. Dentro de este contexto necesitamos replantearnos y cuestionarnos este esquema ideológico político que condiciona las políticas sociales, programas y servicios de bienestar social, así como nuestra acción profesional.

Este es el análisis de la política y las alternativas que propongo para proseguir en la construcción de sociedad, donde nos insertemos en uno de los puntos estratégicos que están moldeando nuestras vidas de manera silenciosa, pero que se siente en las familias, en la escuela, en las calles, convirtiendo a la violencia en normal y aceptada en la cotidianidad de la sociedad puertorriqueña.

\section{Referencias}

Aznar, Hugo. (2005). Ética de la comunicación y nuevos retos sociales. Barcelona: Editorial Paidós Ibérica S.A.

Barbero Jesús Martín. (s.f.): Comunicación Fin De Siglo. ¿Para Dónde Va Nuestra Investigación? Accesado en Internet: Son de Tambora.

Beltrán Luís Ramiro. (2000). Investigación sobre comunicación en Latinoamérica. Inicio, Trascendencia y Proyección. La Paz: Ed. Plural

Brunner, J. y Catalán, C. (1994). Regulación pública de la televisión: Sentido y alcances. Cuadernos de Información, 9, Santiago de Chile. 
Bunge, Mario. (1995). Sistemas sociales y filosofia. Buenos Aires: Editorial Suramericana.

Frederic Jameson. (2003). Consideraciones del CAC sobre la telebasura. Consell de 1'audiovisual de Cataluña. Barcelona: Ed.Sudamericana.

Canino, Maria Josefa y Guardiola, Dagmar. (1995). Formación del Trabajador Social de Puerto Rico. En La Política Social ante los Nuevos Desafios: Cuba y Puerto Rico. San Juan, Puerto Rico: Publicaciones Puertorriqueñas.

Castells, Manuel. (1996). The Rise of the Network Society. Massachussets. Cambridge

Centro Internacional para Periodistas, CIP. (1994). Declaración de Chapultepec. Adoptada por la Conferencia Hemisférica sobre Libertad de Expresión. México: Autor.

Coase, Ronal H. (1994). El Problema del Coste Social. La Empresa, el Mercado y la Ley. Madrid: Alianza Editorial.

Estrada, Aileen. (1995). La construcción social de la realidad y los noticiarios de televisión. Tecne, 2 (1), agosto-diciembre.

Ferrés, Joan. (1996). La televisión subliminal. Socialización mediante comunicaciones inadvertidas. Barcelona: Paidós.

Gordon, A. y Holguín, L. (2005). El Rol de los Medios en la Construcción de Paz: ¿un activo o un pasivo? Consultado en internet, en www.ourmedianet.org/papers/om2003/Holguin_OM3.espan.pdf

Grupo De Defensa Psicológica. (2004). Comités de Usuarios de Radio y Televisión. Consultado en Internet, en http://www.vive.gov.ve/paginas/ comites/comites_usuarios.pdf 
Lara, William. (2004). Despejando dudas sobre la ley venezolana de medios. Consultado en Internet //www.voltairenet.org/article123188.html

Levine, Madelin. (1997). La violencia en los medios de comunicación: cómo afecta al desarrollo de los niños y adolescentes. Bogotá: Norma.

Maturana. León. (2002). Guerra, paz y televisión: una cuestión de paradigmas. Ponencia presentada en el Congreso Iberoamericano de Comunicación y Educación "Luces en el Laberinto audiovisual". Accesado en http:// www.ateiamerica.com/doc/4_36guerra.pdf

National Association of School Psychologists, NASP. (2002). Una Tragedia Nacional: Cómo Promover Tolerancia y Paz en los Niños. Sugerencias para Padres y Escuelas. Accesado en www.naspoline.org.

North, Douglass. (1990). Institutions, Institutional Change and Economic Performance. (Political Economy of Institutions and Decision Series). Cambridge University Press.

Ollivier, Bruno. (1997). Medios y educación: Encuentros del tercer tipo. En $40^{\circ}$ congreso de la AIERI/AIECS. México.

Organización de las Naciones Unidas. (2004) Carta de Derechos en las comunicaciones. New York: Autor.

Osborne, Martin J. (1995). Special models of political competition under plurality rule. Canadian Journal of economics, 27.

Penalva, Clement. (2002). El tratamiento de la violencia en los medios de comunicación: Alternativas. Cuadernos de Trabajo Social, 10, 395-412, Universidad de Alicante.

Piaget, Jean. (1970). El desarrollo mental del niño, en Seis estudios de Psicología. Barcelona: Seix Barral. 
Singer, M.I Slovak, K., Frierson, T., York, P. (1998). Viewing Preferences, Symptoms of Psychological Trauma, and Violent Behaviors Among Children Who Watch Television. CWRU's Mandel School of Applied Social Sciences. Journal of the American Academy of Child \& Adolescent Psychiatry, 37(10), 1041-1048.

Sepúlveda, Héctor. (2006). Suaves Dominaciones. Serie Nueva comunicación. San Juan: Editorial Plaza Mayor.

The TV Parental Guidelines. (2005). About the TV Ratings and V-Chip. Consultado en Internet en www.tvguidelines.org/default.asp

Walsh, D. y Gentile, D (2001). A Validity Test of Movie, Television and VideoGame Ratings. Pediatrics, 107 (6), Junio.

WFMH, World Federation for Mental Health. (2004). Efecto de los Medios de Comunicación sobre el Desarrollo Infantil. Consultado en Internet en www.wfmh.org 
